\title{
Clostridium difficile: epidemiology, diagnostic and therapeutic possibilities-a systematic review
}

\author{
M. Kazanowski $\cdot$ S. Smolarek $\cdot$ F. Kinnarney • \\ Z. Grzebieniak
}

Received: 6 January 2013/ Accepted: 9 October 2013/Published online: 1 November 2013

(C) The Author(s) 2013. This article is published with open access at Springerlink.com

\begin{abstract}
This literature review looks at the epidemiology, clinical manifestations, diagnostics and current medical and surgical management of Clostridium difficile (C. difficile) infection. A literature search of PubMed and Cochrane database regarding $C$. difficile infection was performed. Information was extracted from 43 published articles from 2000 to the present day which met inclusion criteria. C. difficile is a gram-positive, anaerobic bacillus, which is widely found in the environment, especially in the soil. The occurrence of more resistant strains, which is mainly connected with the wide use of antibiotics, resulted in the rapid spread of the bacteria to different hospital departments. Particularly, elderly patients in surgical wards and intensive care units are at significant risk of developing C. difficile infection, which greatly increases morbidity and mortality. Symptoms of infection with $C$. difficile vary greatly. At one end of the spectrum, there are asymptomatic carriers, at the other patients with life-threatening toxic megacolon. Metronidazole is considered to be the drug of choice, but recent guidelines recommend Vancomycin. Fulminant colitis and toxic megacolon warrant surgical intervention. The optimal time for surgery is within $48 \mathrm{~h}$ of initiating conservative treatment without seeing a response, the development of multiple organ failure or a bowel perforation. A factor that has become increasingly important and relevant is the escalating expense of treatment for
\end{abstract}

M. Kazanowski ( $₫) \cdot$ S. Smolarek · Z. Grzebieniak

Second Department of General and Oncological Surgery,

Wroclaw Medical University, Borowska Street 213,

50-556 Wrocław, Poland

e-mail: mikazanowski@gmail.com

F. Kinnarney

Department of General Surgery, St Michaels Hospital,

Dublin, Ireland patients with $C$. difficile infection. It is, therefore, highly recommended to consider reviewing all hospital antibiotic policies and clinical guidelines that may contribute to the prevention of the infection.

Keywords Clostridium difficile .

Pseudomembranous colitis · Fulminant colitis .

Nosocomial infections

\section{Introduction}

The name "Clostridium difficile" $(C$. difficile) comes from the Greek word "Kloster" which means spindle. It was first mentioned in the literature in 1935 by Hall and O'Toole [1]. At first, the bacterium was given the name "Bacillus difficilis." (Latin: difficilis, meaning difficult). This was because of the difficulty encountered isolating the bacteria and also the fact that it had a very slow growth phase during culturing. The name was subsequently changed in the 1970s to C. difficile.

Clostridium difficile is a gram-positive, anaerobic bacillus, which is found widely in the environment, especially in the soil. Despite the fact that even in the first known description of $C$. difficile the authors had mentioned its deadly effects on mice, the complete virulence of the bacterium was not properly recognized until much later. During World War II, Hambre et al. [2] observed using animal models that mice treated for gas gangrene with penicillin suffered from a very severe form of typhlitis. This in fact turned out to be even more deadly than the gangrene itself caused by Clostridium perfringens. This discovery led to new tests in which researchers gave rodents different kinds of antibiotics watching for the development of very similar symptoms. Green [3] used 
guinea pigs in his experiments, during which he was able to induce death by giving them large doses of penicillin. He then studied the stool of the dead guinea pigs and discovered the presence of cytoplasmic changes within. This was the first description of $C$. difficile toxin. It was Cohen and colleagues [4] who actually documented the connection between pseudomembranous colitis and antibiotic therapy. One year after the publication of this association, Tadesco et al. [5] had noticed that patients treated with clindamycin (almost $21 \%$ ) suffered from diarrhea and (10\%) were diagnosed with pseudomembranous colitis. This trial involved over 200 patients and was the first trial in which endoscopy was used so routinely on such a large group of patients. It led to the identification of $C$. difficile as a causative factor for multiple ailments involving the digestive system.

Clostridium difficile is found in $66 \%$ of the digestive tracts of asymptomatic infants and young children. This could be secondary to the fact that not all of the receptors in the intestinal epithelium have matured completely. In adults, colonization affects about $3 \%$ of the population. This number increases considerably during long hospital stays and postoperatively. The bacteria are present mainly in a vegetative form and are very sensitive to atmospheric oxygen. Under the influence of considerable stress, they may take the form of a spore and are thus able to survive harsh environments, such as the acid content of the stomach. With this resilience, $C$. difficile can find itself intact in the small intestine and transform itself back into a vegetative form. It can then colonize the epithelial lining of the mucosa in the digestive tract, and the problems caused by the presence of bacteria are due to several different toxins it produces. The best known are toxin A (enterotoxin) and B (cytotoxin), which under favorable conditions are produced in copious amounts. Inside the cell membrane, these toxins inactivate the transformation pathway mediated by Rho family proteins, which are responsible for the proper construction of actin cytoskeleton and the signal transduction by GTP. This affects the cell and leads to cessation from its regular cycle and apoptosis [6]. Both toxins also affect the strength of the intercellular bonds [7]. The relationship between the amount of toxins in the feces and the severity of symptoms has been demonstrated. Significant increases in toxins in the fecal load are associated with the significant deterioration of the general condition of the patient [8]. Toxin A leads to an increased secretion of fluid within the digestive tract, mucosal inflammation and structural damage. Toxin B is in most cases responsible for the major problems associated with infection. It is estimated that it has approximately 10 times more impact on the gastrointestinal mucosa than toxin A [7]. Brito et al. [7] came to the conclusion that the strains, which do not produce toxin $\mathrm{A}$, are just as dangerous as those which have both toxins. There is also a hypervirulent strain in existence, which was first observed at the beginning of the twenty-first century. This particular strain is responsible for the outbreaks of highly virulent pathogens and is referred to as NAP1/BI/027. The complexity of the name is due to the different methods applied in detecting the presence of the bacteria: pulsed-field gel electrophoresis (NAP1), restriction endonuclease analysis (BI) and polymerase chain reaction (027). Type $\mathrm{C}$ toxin is produced by this particular pathogen. The increasing frequency, with which we now see treatment-resistant and more virulent strains of the bacteria, led the authors of this paper to review the literature on $C$. difficile infection and the treatment options available.

\section{Materials and methods}

A literature was carried out using the electronic databases of PUBMED and Cochrane (up to December 2012) for relevant papers using the following terms: "Clostridium difficile," "Clostridium difficile infection," "Clostridium difficile treatment," "Clostridium difficile colitis" and "Clostridium difficile fecal transplant," limiting our search to only English language articles. The reference lists of used papers were also check and reviewed to identify publications on the same topic.

\section{Results}

Information and data used for this publication were obtained from 43 articles which met the searching criteria.

We excluded search results for which only an abstract was available and case studies; however, we checked the references from those we found to be interesting. We included studies (review papers, meta-analysis and guidelines) which described the epidemiology and first publication about $C$. difficile infections. We compare different papers and results according to the stage and conservative treatment which was used. Data were collected from original papers referring to the possible surgical approaches, those routinely and unusually used.

Data extraction regarding epidemiology, pathophysiology, clinical manifestation with diagnosis and treatment of the infection was completed by the first author and coreviewed by the second author.

Epidemiology

The occurrence of diarrhea and pseudomembranous colitis significantly increased, immediately following the introduction of widely available antibiotic treatments. At first, 
the use of clindamycin was connected with those ailments and following this, the broad use of Penicillin. It took many more years to identify $C$. difficile as being the causative bacterium responsible for most of the symptoms associated with the wide use of antibiotics. The simple way in which the bacteria spread resulted in a significant increase in the number of infections, especially among hospitalized patients. The occurrence of more and more resistant strains resulted in the rapid spread of the bacteria to different departments, particularly the surgical wards and intensive care units but also many other medical wards. In a retrospective Canadian study, diarrhea and pseudomembranous colitis were identified as occurring 4 times more frequently in the general population in 2003 than in 1991 and 10 times more frequently than in 1938 [9]. The same study noted a significant increase in the symptoms of infection among hospitalized patients from 3 to 12 per 1,000 patients (the difference between 1991 and 2003) and up to 43 per 1,000 patients in 2004. Not only has the number of cases grown significantly over the years, but the severity has increased and the general condition of the patient has deteriorated. More and more patients, in addition to standard conservative treatment, require surgery. In another study, $10 \%$ of patients were hospitalized in intensive care units and $2.5 \%$ required an emergency colectomy. The mortality in this study reached $16 \%$ [10]. Similar statistics were observed in Europe and the USA, where in 1999-2007, C. difficile was the main contributor to death in patients with inflammatory bowel disease [11].

Nosocomial infections are characterized by a much more severe form of the disease than in the general population, and a higher incidence of $C$. difficile infection has been noted, especially since 2000 . This problem becomes particularly apparent among the patients aged over 65 years. According to different authors, the frequency of the carrier stage in patients with long hospital stays or those treated in the intensive care units ranges from 20 to $50 \%$, while in healthy adults, this quantity reaches only about $3 \%$ [12]. Infected patients are often asymptomatic and although they may not feel any discomfort, they act as a reservoir for the bacteria and facilitate the spread of the pathogen among other patients. Infection spreads easily by the fecal-oral route and by direct contact with the patient (fomites), in particular through the hands of hospital personnel, clothes and stethoscopes. Patients, who during their hospital stay were already carriers, usually experience a much milder form of infection or remain asymptomatic [13]. The major risk factor for $C$. difficile infection is widespread use of antibiotics, often without the appropriate indications (Table 1). The use of antibiotics disrupts the natural colonic flora, thereby providing $C$. difficile with the opportunity to multiply and produce its toxins. Other risk
Table 1 Antibiotic groups that may predispose to $C$. difficile infection

\begin{tabular}{lll}
\hline Commonly & Occasionally & Seldom \\
\hline Fluoroquinolones & Macrolides & Aminoglycosides \\
Clindamycin & Trimethoprim & Tetracyclines \\
Penicillins & Sulfonamides & Chloramphenicol \\
Cephalosporins & & Metronidazole \\
& & Vancomycin \\
\hline
\end{tabular}

factors include long duration of admission, advanced age, severe comorbidities, the use of proton pump inhibitors, enteral feeding, gastrointestinal surgery, chemotherapy and the use of tumor suppressor agents in postoperative transplant patients [14].

\section{Clinical presentations}

Symptoms of infection with $C$. difficile are very diverse. At one end of the spectrum, there are asymptomatic carriers at the other patients with life-threatening toxic megacolon. Infection and colonization itself are not the only prerequisite for the development of severe symptoms. What is necessary is a major disturbance of the internal bacterial flora, the risk factors which have already been mentioned and the use of antibiotics, which all have an important role in altering the intestinal flora.

\section{Carrier stage}

This spreads very quickly in the hospital environment, in particular among patients treated in the intensive care units and surgery departments. Most asymptomatic cases progress without any clinical manifestations. McFarland et al. [15] studied 428 patients admitted to hospital within an 11-month period. Twenty-one percent of patients, who were negative for $C$. difficile infection prior to hospitalization, were then subsequently indentified as asymptomatic carriers. Sixty-three percent remained carriers of the infection until the end of the study. According to Lawrence [16], as many as $50 \%$ of hospitalized patients who have had no prior contact with the pathogen become carriers following lengthy hospital stays. Disruption of normal intestinal flora can easily occur with the use of antibiotics and the proliferation of bacteria within the gastrointestinal tract, leaving the intestine susceptible to the adverse influence of bacterial toxins. As previously mentioned, in the carrier stage of $C$. difficile, the severity and frequency of symptoms of the disease are limited. Research regarding the treatment of carriers currently is scarce and is not considered to be of benefit. Treatment of asymptomatic carriers is not recommended [16]. 


\section{C. difficile-associated diarrhea (CDAD)}

This bacterium is responsible for the majority of the cases of diarrhea in hospitals. Its presence is significantly higher in the hospital environment, where therapies that are used require a different group of antibiotics, such as clindamycin, cephalosporins, fluoroquinolones and penicillins [17]. Diarrhea usually occurs after $48-72 \mathrm{~h}$ after infection and is often accompanied by severe abdominal pain and cramps. There can be 10-15 bowel movements a day. A significant number of stools per day can lead to changes in the electrolyte and water balance. In patients with severe conditions, especially after surgery, CDAD increases mortality and morbidity rates.

\section{C. difficile-associated colitis (CDAC)}

Symptoms are very similar to those found in CDAD but also include pyrexia and leukocytosis (with an average white blood cell count of $15 \times 10^{9} / \mathrm{L}$ ). Regarding the physical examination, the most commonly elicited sign in CDAC is abdominal guarding. Other findings can include significant levels of dehydration and a positive fecal occult blood test. A colonoscopy can be helpful at an early stage as specific distinctive changes will be visible in the wall of the bowel. These include characteristic erythematous mucosa with noted friability and bleeding on contact (Figs. 1, 2). Research from Wanahita et al. documents that out of 60 patients with unexplained leukocytosis, $58 \%$ had stool cultures positive for the bacterial toxins. In such cases, symptoms of diarrhea were observed approximately 24-48 h later [18]. Therefore, in cases of patients receiving antibiotics who have a high white blood cell count (WBC), even in the absence of diarrhea, $C$. difficile infection should be suspected.

\section{Pseudomembranous colitis}

This is the most well-known form of $C$. difficile infection. During endoscopy, a characteristic yellow plaque can be observed in the mucosa of the colon and sometimes in the terminal ileum, which forms the basis for early diagnosis (Fig. 3). These plaques are small ulcerations of the mucous membranes, which trigger the release of serum proteins, mucus and inflammatory cells [6] (Fig. 4). Lymphocytes are found in biopsies of the lesion(s), and the patient may be classified into one of the three groups depending on the severity of infection (Table 2). Accompanying symptoms include severe abdominal pain, dehydration and often hypoalbuminemia $(<30 \mathrm{mg} / \mathrm{L})$. It is essential to initiate the appropriate medical treatment for patients with pseudomembranous colitis due to the potential toxic effects of the infection.

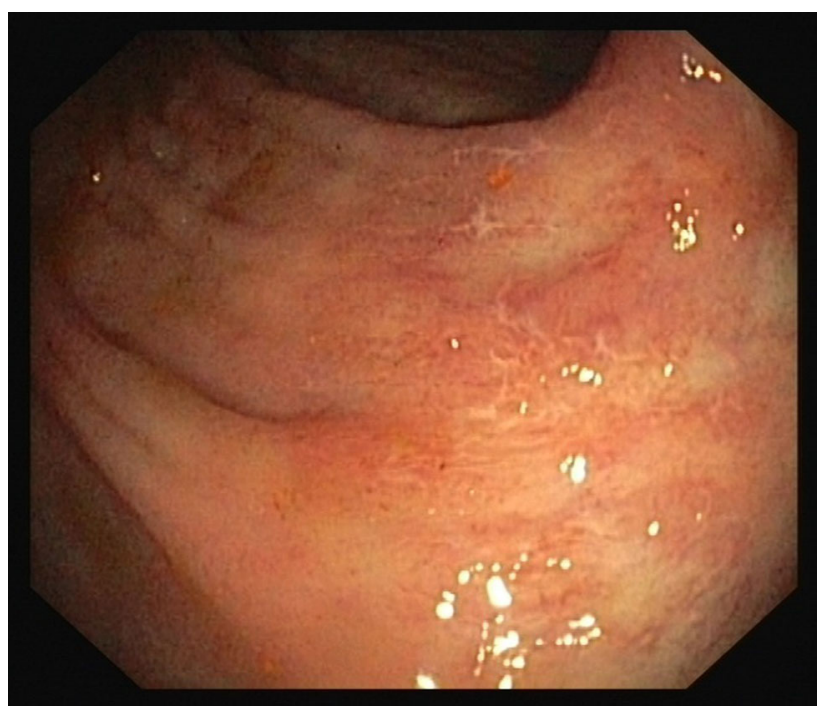

Fig. 1 C. difficile colitis-picture 1 minor changes

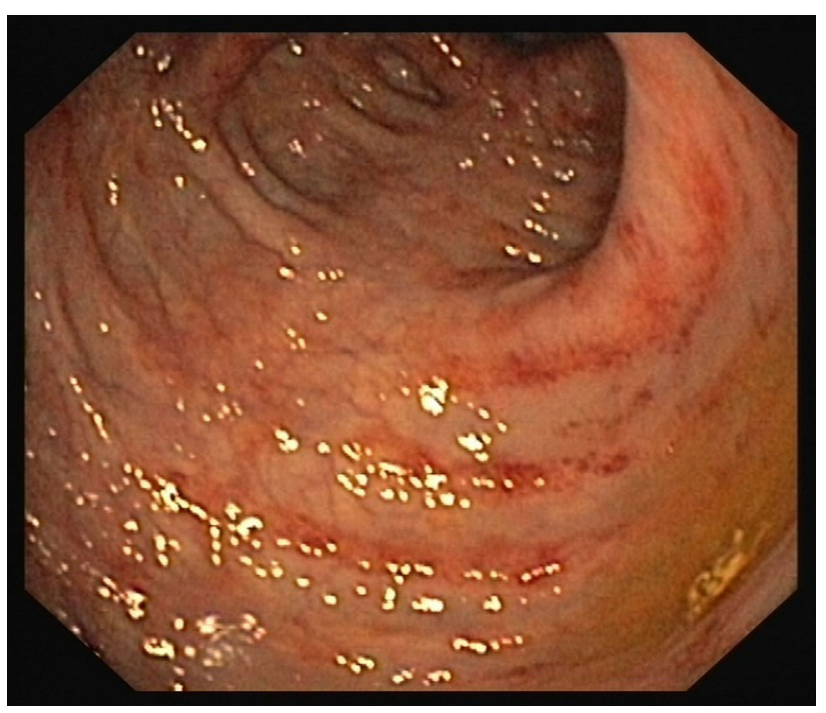

Fig. 2 C. difficile colitis-picture 2 minor changes

Relapses occur in about 10-25\% of cured patients. Frequently, re-infection can be much more severe, and there is a greater predisposition to subsequent episodes of pseudomembranous colitis [19].

\section{Fulminant colitis}

Generally, the natural progression of colitis allows one to conceive and follow a treatment plan; however, sometimes a fulminating form can develop. This form of inflammatory bowel disease develops only in 3-8\% of the patients [17]. A significant rise has been noted in recent years and is associated with a hypervirulent strain of the bacteria. This strain leads to the development of more systemic 


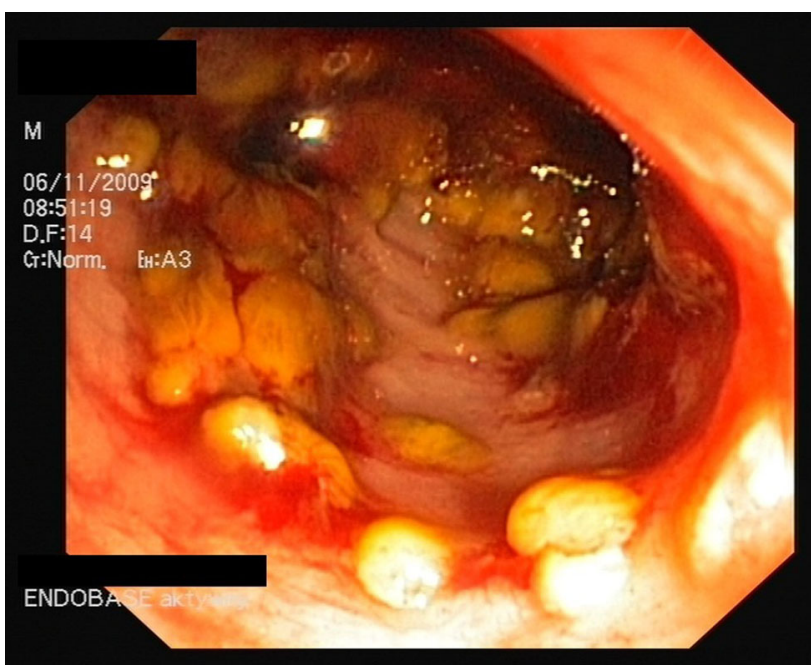

Fig. 3 C. difficile pseudomembranous colitis

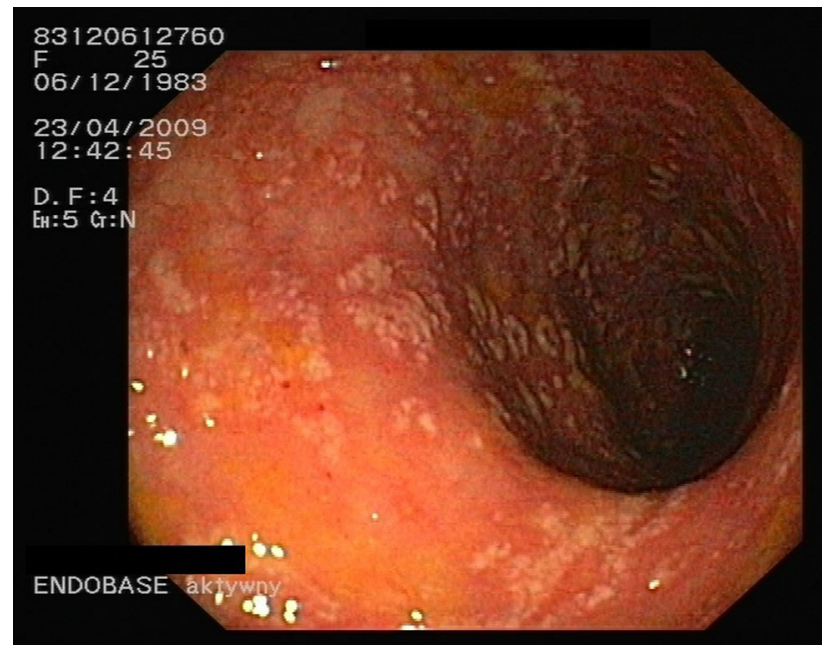

Fig. 4 C. difficile pseudomembranous colitis

symptoms, multiple organ failure and overall increased mortality. Findings during physical examination include involuntary abdominal guarding. Full blood count analysis tends to show a marked leukocytosis $\left(40 \times 10^{9} / \mathrm{L}\right.$ or more $)$ [18] and anemia secondary to bleeding from gastrointestinal tract ulcers. Diarrhea can vary significantly, depending on the course of the disease, from a few episodes per day to complete obstruction and dilatation of the gastrointestinal tract. In the latter case, emergency surgical intervention is required as the mortality rate in these patients is very high and can reach $60 \%$ [20], especially in older patients. If an inpatient has no history of $C$. difficile infection, diagnostic tests of the colon are still necessary. Stool cultures should be obtained. Colonoscopy should be performed by an experienced endoscopist in order to
Table 2 Histopathologic severity of pseudomembranous colitis

\begin{tabular}{|c|c|}
\hline Classification & Description of changes \\
\hline Type 1 & $\begin{array}{l}\text { Mildest form, most of the changes are limited only to } \\
\text { the superficial epithelium. Pseudomembranous } \\
\text { changes are present, but ulcers are found only } \\
\text { occasionally }\end{array}$ \\
\hline Type 2 & $\begin{array}{l}\text { More evident changes in the colonic mucosa, gland } \\
\text { disorders and significantly increased amount of } \\
\text { secreted mucus. Inflammation that invades the } \\
\text { basement membrane }\end{array}$ \\
\hline Type 3 & $\begin{array}{l}\text { Full thickness necrosis is noticed within the whole } \\
\text { bowel wall with pseudomembranous changes }\end{array}$ \\
\hline
\end{tabular}

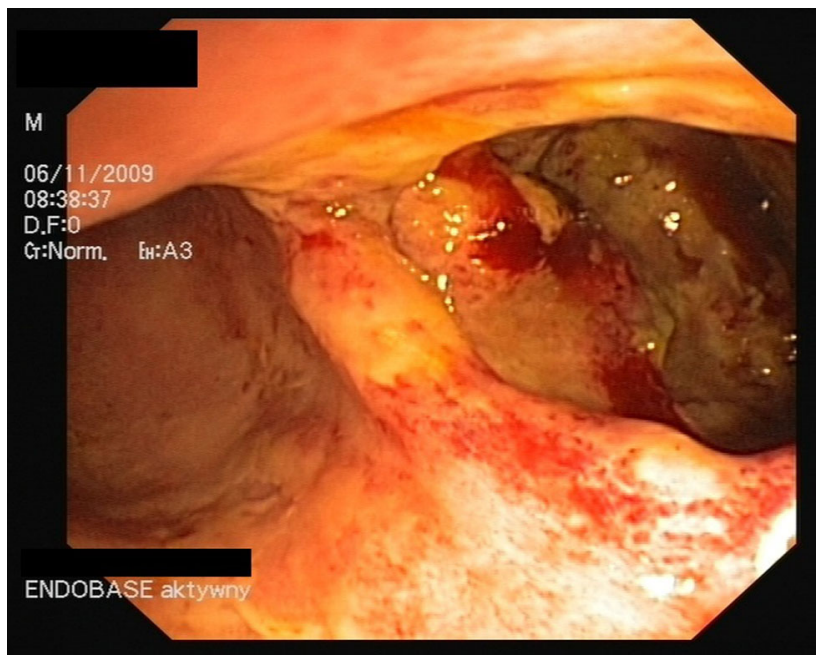

Fig. 5 Perforation in C. difficile colitis

minimize the volume of air blown into the colon. Due to the risk of perforation, many doctors are wary of performing a colonoscopy during an acute episode of colitis. In fact, complications are quite rare (Fig. 5). The purpose of performing an urgent colonoscopy is not to evaluate the entire colon, but only to visualize the lining of the rectum and the distal colon. If obvious features indicative of fulminant colitis are found during the procedure, the endoscopist can simply remove the scope without having to examine the entire bowel [21].

\section{C. difficile-associated enteritis}

Inflammation of the small intestine in the course of C. difficile infection is quite rare. However, when it does occur, it tends to be seen in patients postcolectomy and in those with end ileostomies where high outputs are observed. In elderly patients who have multiple comorbidities, treatment in special referral centers is advisable [22]. 


\section{Appendicitis}

Appendicitis in the course of $C$. difficile infection is very rare. Up until 2007, only three cases have been described in the literature. However, the authors suggest that this complication may be significantly underdiagnosed, as many of the cases may have responded well to the conservative treatment, and no histology samples/specimens were available [23].

\section{Diagnosis}

Diagnostic evaluation of stool samples should be carried out in patients with $C$. difficile infection who suffer from clinically significant diarrhea, i.e., 3 or more episodes of loose stool per day for 2 or more days. Other signs associated with diarrhea such as pyrexia and leukocytosis are suggestive of the diagnosis.

\section{Laboratory tests}

The easiest test is to detect the presence of toxins A and B in the stool sample. An enzyme immunoassay (EIA) has specificity up to $95 \%$, and the result is available after $4 \mathrm{~h}$. However, the sensitivity is significantly reduced to $70-80 \%$ due to the large number of false negatives [24]. The most reliable test is a stool culture. The sensitivity of this test reaches $90 \%$, but the results are not available for approximately 4-5 days. In addition to this time delay, not all laboratories routinely perform this assay. There are some molecular techniques available to identify the presence of the genome and its replication. These include polymerase chain reaction (PCR) ribotyping (a popular method because of its availability, efficiency and high specificity up to $97 \%$ and sensitivity close to $91 \%$ ), pulsed-field gel electrophoresis (PFGE), multilocus variable number tandem repeat analysis (MLVA) and finally multilocus sequence typing (MLST) [25].

\section{Endoscopy}

Colonoscopy and sigmoidoscopy are both valuable diagnostic tools in diagnosing $C$. difficile. In the case of pseudomembranous colitis, the visible findings typically include a characteristic yellow plaque in the intestinal mucosa with ulceration and some associated bleeding. Table 3 shows the 4 main indications for colonoscopy according to Hookman'a et al. [26]. In a patient with the classical clinical symptoms and a positive stool culture, the endoscopic examination may be waived. In the case of fulminant colitis, it should, however, be performed, taking special care not to cause perforation.
Table 3 Indications for colonoscopy in the diagnosis of $C$. difficile infection

Indications for diagnostic colonoscopy

1. The results of the laboratory tests are negative, but there is a high probability of infection due to clinical symptoms

2. Earlier diagnosis required before the results of laboratory tests

3. Failure to respond to treatment with antibiotics

4. Atypical disease with obstruction and mild diarrhea

Imaging studies

A computed tomography (CT) scan of the abdomen and pelvis may be helpful in the advanced stages, when the wall of the intestine is characteristically thickened. Images are not specific for bacterial infections [27].

\section{Treatment}

Clostridium difficile infection is one of the most common causes of nosocomial infections and in particular is responsible for increased morbidity and mortality in elderly patients. The bacteria colonize the gastrointestinal tract, when the physiological bacterial flora is disrupted due to the use of antibiotics. Treatment of the infection depends on the severity of the disease and the symptoms. It can be treated conservatively or with surgery.

The most important initial treatment step is to cease administration of the antibiotic that caused the $C$. difficile infection (CDI). The continuous administrations of antibiotics, which do not treat $C$. difficile, not only worsen the patient's condition, but may also affect their susceptibility to re-infection [28]. If, due to the primary disease, administration of antibiotics is required, it would be prudent to incorporate antibiotics that are less responsible for extending CDI, such as aminoglycosides, sulfonamides, macrolides, tetracyclines and vancomycin. The general principles of prevention of further infections should also be kept in mind. Patients with suspected or confirmed CDI should be placed in isolation because of the high risk of contamination. All medical staff should perform thorough hand hygiene following contact with patients and also sanitize any medical equipment used, e.g., stethoscope. Patients should be well hydrated, and their electrolyte levels should be carefully monitored. There are no dietary limitations with the exception of patients who have surgery already scheduled. In patients with typical symptoms of CDI, such as diarrhea, abdominal pain, nausea and positive stool cultures, antibiotics should be initiated [29]. Empirical therapy is only indicated when there is a very high probability of infection and while awaiting the results of diagnostic tests. Carriers should not be treated with antibiotics when there are no clinical symptoms of the infection. 
In the treatment of less severe infections, initial therapy should consist of metronidazole or vancomycin. Several randomized clinical trials showed significant efficacy of these antibiotics in the treatment of CDI [30]. Both drugs in a study by Zar and colleagues [30] showed similar efficacy (90-98 \%), but metronidazole is considered to be the drug of choice. The advantages of metronidazole are much lower costs of the therapy and reduced spread of vancomycin-resistant enterococci (VRE). Metronidazole should be prescribed at a dose of $500 \mathrm{mg} 3$ times daily or $250 \mathrm{mg}$ 4 times a day for 10-14 days. The oral dose of vancomycin is $125 \mathrm{mg}$ every $6 \mathrm{~h}$. The use of higher doses such as $500 \mathrm{mg}$ has no documented association with a shorter recovery time. Intravenous forms of the medication are not used as they have poor penetration into the gastrointestinal tract [31]. Further examination of stool samples during treatment is not indicative of response to treatment as approximately $50 \%$ of patients may have positive results up to 6 weeks after cessation of treatment [31]. The use of monoclonal antibodies against toxins $\mathrm{A}$ and $\mathrm{B}$ is a subject of a great interest and hope. Currently they are not used or recommended for routine treatment. Lowy et al. [32] studied 200 patients to whom standard doses of antibiotics were given (101 in the antibody group and 99 in the placebo group). The incidence of relapse was 7-25\%. More randomized trials are needed to determine the benefit of this therapy as a standard procedure.

Patients with severe infections may develop systemic failure with copious diarrhea and must be treated in the intensive care unit or surgical ward. There is no general classification of "severity of infection" available, but in the literature, a number of signs and symptoms are reported that indicate which patients are candidates for intensive therapy. These include white count $\mathrm{C}>15 \times 10^{9} / \mathrm{L}$, elevated creatinine, temperature $>38.8{ }^{\circ} \mathrm{C}$ and albumin $<2.5 \mathrm{mg} / \mathrm{dL}$. Generally, the physicians clinical decision and opinion are considered to be the most important when initiating intensive treatments. Recent guidelines from 2010 [33] recommend vancomycin as the drug of choice. Its main advantage is that vancomycin given orally is not absorbed by the body so the full dose reaches the large intestine, the site of infection. Many clinicians use Vancomycin per os at a dose of $500 \mathrm{mg} 4$ times daily despite a lack of evidence for the effectiveness of this therapy. The recommended dose is still $125 \mathrm{mg} 4$ times daily [33]. In patients refractory to this treatment, fidaxomicin or metronidazole can be used. In patients with a bowel obstruction, vancomycin enemas with continuous oral [34] or intravenous therapy, plus metronidazole every $8 \mathrm{~h}$ is recommended.

Patients with severe and protracted infections can sometimes develop rectal toxicity (toxicum megacolon), perforation and necrosis of the intestine or rapidly progressive infections with multiple organ failure. These patients require surgical intervention [35]. The optimal time for surgery is within $48 \mathrm{~h}$ of initiating conservative treatment without seeing a response, the development of multiple organ failure or a bowel perforation [20]. The difficulty is in determining the optimal time for surgical intervention as not all patients will survive the initial $48 \mathrm{~h}$. Considerations also need to be made regarding cases of bowel obstruction and persistent diarrhea and vomiting which are not suitable for conservative treatment. The Canadian retrospective study already referred to [21] showed that a colectomy was the most beneficial treatment in patients above 65 years of age, with a WBC $>20 \times 10^{9} / \mathrm{L}$ and elevated serum lactate between 2.2 and $4.9 \mathrm{mmol} / \mathrm{L}$. Positive peritoneal signs, obstruction, perforation and signs of toxic megacolon should also be included in these criteria.

CDI infections are currently treated by two different surgical approaches. One is subtotal colectomy. This involves the removal of the entire colon with the creation of an ileostomy, leaving the rectum in place. The other procedure is less invasive - a diverting loop ileostomy with colonic lavage.

\section{Subtotal colectomy}

The number of subtotal colectomies performed continues to escalate due to the presence of hypervirulent strains of $C$. difficile. Currently, about $5 \%$ of patients infected with $C$. difficile reach the stage of fulminant colitis and undergo surgery. The procedure selected and the outcome depends on the level of experience of the surgeon, but better results are obtained in the case of total colectomy [36]. Earlier surgical intervention is also associated with better results. An emergency colectomy for advanced forms of $C$. difficile is associated with higher mortality rates. Al-Abed et al. [37] operated on $3.7 \%$ of his patients with an associated mortality rate of over $40 \%$. The majority of patients who had significant comorbidities $(75 \%)$ did not survive after an emergency colectomy. Anton D. Parera et al. [38] had very similar results. The 30-day mortality rate was $45.7 \%$. Therefore, it is crucial to identify infected patients early before they progress into fulminant colitis and organ failure.

\section{Diverting loop ileostomy with colonic lavage}

This procedure may be an interesting alternative to a colectomy. Neal et al. [39] operated on 42 patients using this procedure. Comparing colectomy and diverting loop ileostomy with lavage, the authors noted a reduction in mortality from 50 to $19 \%$. After creating the ileostomy, the colon may be flushed with warm polyethylene glycol. Postoperatively vancomycin enemas can be administered 
via the ileostomy. This procedure was performed laparoscopically in 32 patients, which was equally effective and less harmful to the patient at the same time. This is especially important among elderly patients.

\section{Fecal transplant}

As previously mentioned, a disruption in the balance of the normal intestinal flora is a major risk factor for $C$. difficile infection and indeed recurrent infections. In several uncontrolled trials, the administration of stool from a healthy donor has been used with a high degree of success [40]. If a "fecal transplant" is considered, the donor should be screened for transmissible diseases. Logistic issues to be considered include timing, collection and processing of the specimen from the donor. The feces can be delivered via nasogastric tube or enema.

\section{New treatments}

Some new, interesting therapies are being tried and tested in C. difficile treatment. Vancomycin therapy followed by rifaximin may be effective in the treatment of $C$. difficile infection. In one series, 8 patients received a 2 -week course of rifaximin once they were clinically asymptomatic, after the last administration of vancomycin. Seven patients had no further recurrence of infection [41]. Also, intravenous immunoglobulin (IVIG) containing $C$. difficile antitoxin has been used in some patients with severe $C$. difficile colitis. A retrospective review with a comparison to some case reports revealed that there is no significant difference in the clinical outcomes [42]. Probiotics may also be effective in the prevention and treatment of CDAD, in several ways: the alteration of intestinal flora, increased antimicrobial activity, intestinal barrier protection and immunomodulation. The clinical role of this therapy is an evolving area of study [43].

\section{Discussion}

In recent years, the number of nosocomial infections has risen significantly. This is almost certainly because of the overprescribing of antibiotics and one could certainly question the clinical indications for said use. Also to blame are the lengthy admission stays in surgical departments and intensive care units. The demographics of the surgical patient have changed in recent times, and there are now more elderly and medically complex patients. Several years ago, this subset of the population may have been deemed unsuitable for surgical intervention and therefore exempt from procedures. As we now routinely treat an increasingly elderly subset of patients, we have to allow for the fact that their average length of hospital stay is longer than that of other patients. This group would realistically stay more than 7 days as inpatients, thereby running a major risk of contracting a $C$. difficile infection. In these cases, preventative measures, a heightened level of awareness and knowledge of early clinical symptoms are of vital importance.

Obviously with the ever increasing severity of the infection comes an associated increase in morbidity and mortality. There are numbers of independent predictors of mortality for CDAI (Table 4). Patients, who present with these risk factors, especially with strong predictors, should have early surgical consultations and early aggressive surgical intervention should be considered.

The choice of initial treatment, surgical versus medical, and the type of surgical resection influence the final outcomes (Table 5).

If we take into account the lack of specific guidelines for the treatment of $C$. difficile infection, and the number of currently available surgical techniques, physicians and surgeons have a considerable range of potential approaches to use. Figure 6 shows the clinical approach to treatment of C. difficile infection.

Table 4 Predictors of mortality for fulminant $C$. difficile colitis

Strong predictors of mortality for fulminant $C$. difficile colitis

1. Age $\geq 70$ years

2. Severe infection $\mathrm{WBC} \geq 35,000$ or $\leq 4,000 / \mu \mathrm{L}$ or neutrophil bands $\geq 10 \%$

3. Need for cardiorespiratory support (vasopressin or intubation)

4. Arterial lactate $>4.9$

5. Mental status change

Weak predictors of mortality for fulminant $C$. difficile colitis

1. Type of surgery (total colectomy vs. segmental resection)

2. Delayed surgical intervention

3. Admission to other than surgical ward

4. Multiple comorbidities

5. No vancomycin use during medical treatment

$W B C$ white cell count

Table 5 Morbidity related to $C$. difficile infection and treatment

Morbidity after fulminant $C$. difficile colitis (\%)

Overall 30-day mortality

34-57

5-Year survival rates

16.3-38

Subtotal colectomy and end ileostomy mortality rate 11

Segmental colectomy mortality rate $\quad 42-100$

Diverting loop ileostomy with colonic lavage mortality rate 19

Stoma reversal rate

20 


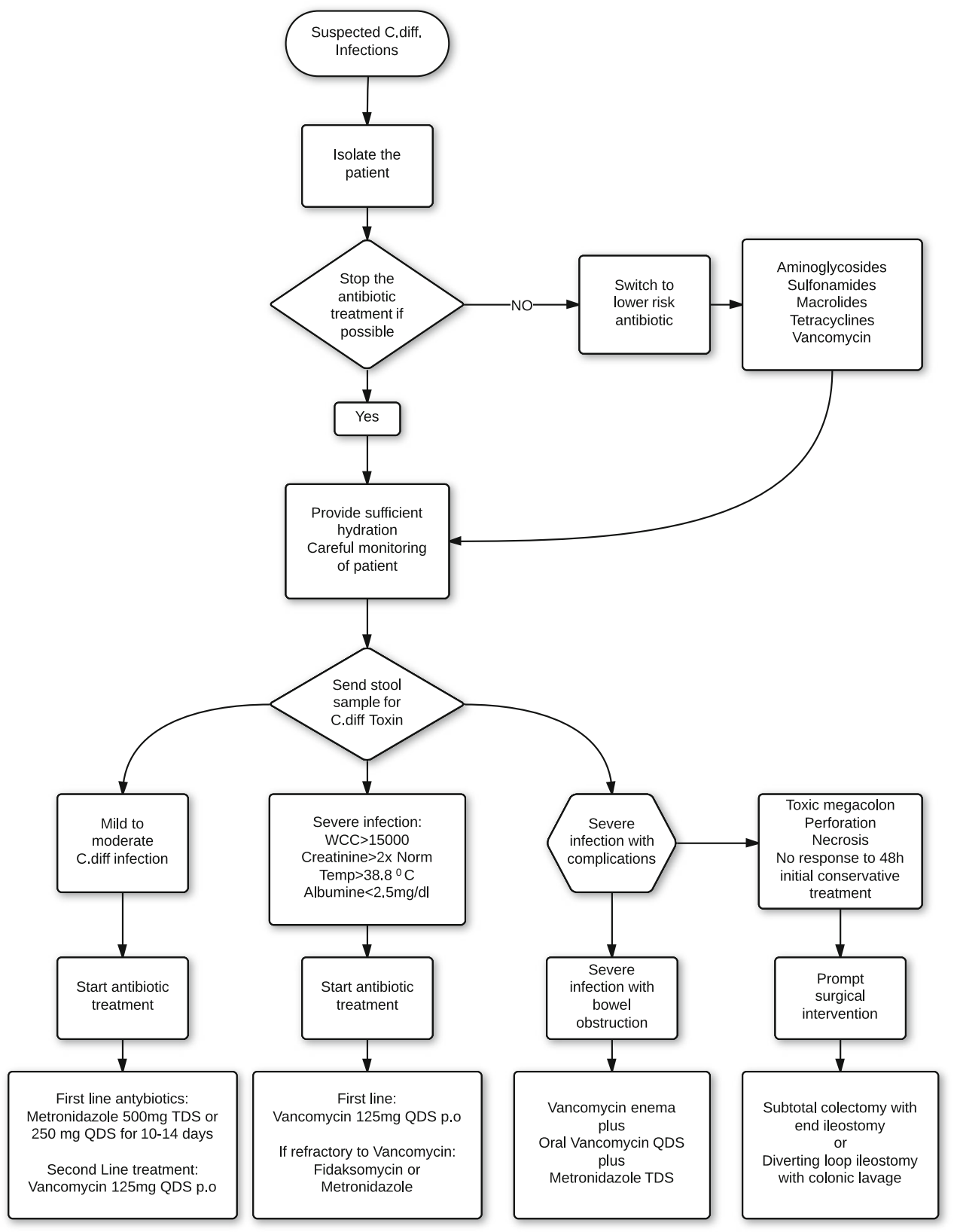

Fig. 6 Clinical approach to treatment of $C$. difficile infection

\section{Conclusions}

Despite the overall positive results following a subtotal colectomy, recent research shows that there is potential to develop safer and less invasive techniques. The authors of this study would like to highlight the ever increasing and problematic issue of rising levels of nosocomial infections. Further research is of paramount importance to help reduce their occurrence.

\section{Conflict of interest None}

Open Access This article is distributed under the terms of the Creative Commons Attribution License which permits any use, distribution, and reproduction in any medium, provided the original author(s) and the source are credited.

\section{References}

1. Hall C, O'Toole E (1935) Intestinal flora in newborn infants with a description of a new pathogenic anaerobe, Bacillus difficilis. Am J Dis Child 49:390-402 
2. Hambre M, Rake G, McKee M et al (1943) The toxicity of penicillin as prepared for clinical use. Am J Med Sci 206:642-652

3. Green RH (1974) The association of viral activation with penicillin toxicity in guinea pigs and hamsters. Yale J Biol Med 47:166-181

4. Cohen E, McNeill J, Wells F (1973) Clindamycin-associated colitis. J Am Med Ass 223:1379-1380

5. Tadesco J, Barton W, Alpers H (1974) Clindamycin-associated colitis: a prospective study. Ann Intern Med 81:429-433

6. Riegler M, Sedivy R, Pothoulakis C et al (1995) Clostridium difficile toxin $\mathrm{B}$ is more potent than toxin $\mathrm{A}$ in damaging human colonic epithelium in vitro. J Clin Invest 95:2004-2011

7. Brito A, Sullivan W, Ciesla P et al (2002) Clostridium difficile toxin A alters in vitro-adherent neutrophil morphology and function. J Infect Dis 185:1297-1306

8. Akerlund T, Svenungsson B, Lagergren A et al (2006) Correlation of disease severity with fecal toxin levels in patients with Clostridium difficile-associated diarrhea and distribution of PCR ribotypes and toxin yields in vitro of corresponding isolates. J Clin Microbiol 44:353-358

9. Pepin J, Valiquette L, Alary E et al (2004) Clostridium difficileassociated diarrhea in a region of Quebec from 1991 to 2003: a changing pattern of disease severity. CMAJ 171:466-472

10. Pepin J, Valiquette L, Cossette B (2005) Mortality attributable to nosocomial Clostridium difficile-associated disease during epidemic caused by a hypervirulent strain in Quebec. CMAJ 173:1037-1042

11. Hall J, Curns T, McDonald C, Parashar UD, Lopman BA (2012) The roles of Clostridium difficile and norovirus among gastroenteritis-associated deaths in the United States, 1999-2007. Clin Infect Dis 55:216-223

12. Riggs M, Sethi K, Zabarsky F et al (2007) Asymptomatic carriers are a potential source for transmission of epidemic and nonepidemic Clostridium difficile strains among long-term care facility residents. Clin Infect Dis 45:992-998

13. Shim K, Johnson S, Samore H et al (1998) Primary symptomless colonization by Clostridium difficile and decreased risk of subsequent diarrhea. Lancet 351:663-666

14. Loo G, Bourgault M, Poirier L et al (2011) Host and pathogen factors for Clostridium difficile infection and colonization. N Eng J Med 365:1693-1703

15. McFarland V, Mulligan E, Kwok Y et al (1989) Nosocomial acquisition of Clostridium difficile infection. N Engl J Med 26:204-210

16. Lawrence J (2007) Contemporary management of Clostridium difficile-associated disease. Gastroenterol Endosc News Speed 5:35-40

17. Adams D, Mercer W (2007) Fulminant Clostridium difficile colitis. Curr Opin Crit Care 13:450-455

18. Wanahita A, Goldsmith A, Marino J et al (2003) Clostridium difficile infection in patients with unexplained leukocytosis. Am J Med 115:543-546

19. Fekety R, McFarland V, Surawicz M et al (1997) Recurrent Clostridium difficile diarrhea: characteristics of and risk factors for patients enrolled in a prospective, randomized, double-blinded trial. Clin Infect Dis 24:324-333

20. Dallal M, Harbrecht G, Bojoukas J et al (2001) Fulminant Clostridium difficile: an underappreciated and increasing cause of death and complications. Ann Surg 235:363-372

21. Lamontagne F, Labbé AC, Haeck O et al (2007) Impact of emergency colectomy on survival of patients with fulminant Clostridium difficile colitis during an epidemic caused by a hypervirulent strain. Ann Surg 245:267-272

22. Vesoulis Z, Williams G, Matthews B (2000) Pseudomembranous enteritis after proctocolectomy: report of a case. Dis Colon Rectum 43:551-554

23. Brown A, Rajappannair L, Dalton B et al (2007) Acute appendicitis in the setting of Clostridium difficile colitis: case report and review of the literature. Clin Gastroenterol Hepatol 9:969-971

24. Turgeon K, Novicki J, Quick J et al (2003) Six rapid tests for direct detection of Clostridium difficile and its toxin in fecal samples compared with the fibroblast cytotoxicity assay. J Clin Microbiol 41:667-670

25. Kachrimanidou M, Malisiovas N (2011) Clostridium difficile infection: a comprehensive review. Crit Rev Microbiol 37:178-187

26. Hookman P, Barkin S (2009) Clostridium difficile associated infection, diarrhea and colitis. World J Gastroenterol 15:1554-1580

27. Bartlett G (2002) Clinical practice. Antibiotic-associated diarrhea. N Engl J Med 346:334-349

28. Hu Y, Katchar K, Kyne L et al (2009) Prospective derivation and validation of a clinical prediction rule for recurrent Clostridium difficile infection. Gastroenterology 136:1206-1214

29. Drekonja M, Butler M, MacDonald R et al (2011) Comparative effectiveness of Clostridium difficile treatments: a systematic review. Ann Intern Med 155:839-847

30. Zar A, Bakkanagari R, Moorthi M et al (2007) A comparison of vancomycin and metronidazole for treatment of Clostridium difficile-associated diarrhea, stratified by disease severity. Clin Infect Dis 45:302-307

31. Fekety R, Silva J, Kauffman C et al (1989) Treatment of antibiotic-associated Clostridium difficile colitis with oral vancomycin: comparison of two dosage regimens. Am J Med 86:15-19

32. Lowy I, Molrine DC, Leav BA et al (2010) Treatment with monoclonal antibodies against Clostridium difficile toxins. N Engl J Med 362:197-205

33. Cohen H, Gerding N, Johnson S et al (2012) Clinical practice guidelines for Clostridium difficile infection in adults: 2010 update by the society for healthcare epidemiology of America (SHEA) and the infectious diseases society of America (IDSA). Infect Control Hosp Epidemiol 31:431-455

34. Apisarnthanarak A, Razavi B, Mundy M (2002) Adjunctive intracolonic vancomycin for severe Clostridium difficile colitis: case series and review of the literature. Clin Infect Dis 35:690-696

35. Sailhamer A, Carson K, Chang Y et al (2009) Fulminant Clostridium difficile colitis: patterns of care and predictors of mortality. Arch Surg 144:433-439

36. Butala P, Divino D (2010) Surgical aspects of fulminant Clostridium difficile colitis. Am J Surg 200:131-135

37. Al-Abed Y, Gray E, Rothnie N (2010) Outcomes of emergency colectomy for fulminant Clostridium difficile colitis. Surgeon $8: 330-333$

38. Perera A, Akbari R, Cowher M (2010) Colectomy for fulminant Clostridium difficile colitis: predictors of mortality. Am Surg 76:418-421

39. Neal D, Alverdy C, Hall E et al (2011) Diverting loop ileostomy and colonic lavage: an alternative to total abdominal colectomy for the treatment of severe, complicated Clostridium difficile associated disease. Ann Surg 254:423-427

40. Gustafsson A, Lund-Tonnesen S, Berstad A et al (1998) Faecal short-chain fatty acids in patients with antibiotic-associated diarrhea, before and after faecal enema treatment. Scand J Gastroenterol 33:721-727

41. Garey W, Ghantoji S, Shah N et al (2011) A randomized, doubled-blind, placebo-controlled pilot study to assess the ability of rifaximin to prevent recurrent diarrhea in patients with Clostridium difficile infection. J Antimicrob Chemother 66:28502855

42. Juand P, Skledar J, Zgheib K et al (2007) Clinical outcomes of intravenous immune globulin in severe clostridium difficileassociated diarrhea. Am J Infect Control 35:131-137

43. McFarland V (2006) Meta-analysis of probiotics for the prevention of antibiotic associated diarrhea and the treatment of Clostridium difficile disease. Am J Gastroenterol 101:812-822 